\title{
STYLE ET REGISTRE DE LANGUE EN TRADUCTION
}

\author{
Raluca-Nicoleta BALAȚCHI \\ Université Ştefan cel Mare, Suceava
}

\begin{abstract}
En): In this paper, we seek to assess the difficulties of translating register in literary discourse, on the basis of a corpus constituted by the translation and retranslation of Marguerite Duras' Moderato Cantabile into Romanian. Translating register is considered by most Translation Studies scholars as a primary criterion in assessing the quality of a translation. To what extent can the translator preserve the register features when rendering the text into the target language? How far do his/her subjective choices influence the cultural identity of the translated text? Does the practice of retranslation bring essential changes from this point of view?
\end{abstract}

Keywords (En): Translation ; Translating register ; Literary discourse ; Retranslation ; Marguerite Duras

\section{Pour introduire notre propos}

La question des registres et niveaux de langue, dont l'utilité dans le processus de compréhension et de description des productions discursives, à l'oral comme à l'écrit, n'est plus à démontrer, a une longue tradition dans l'histoire de la linguistique, remontant, comme le précise GADET (1996: 18), à la rhétorique grecque, où l'on faisait la distinction entre les différents tons (sublime, moyen, bas).

L'analyse de ce phénomène qui affecte la plupart des niveaux de l'organisation langagière a imposé, au fil du temps, et en fonction des différentes approches, une reconsidération des catégories et des instruments méthodologiques. De ce point de vue, il est important de mentionner le rôle des études sur des corpus attestés, en particulier sur le français parlé.

En raison de sa complexité, le registre de langue pose des problèmes majeurs en traduction, comme le soulignent les traductologues et comme en témoignent les traducteurs de différents types de discours. L'intérêt pour la notion de niveau en tant que telle est partiellement dû aux réflexions de la stylistique comparée (cf. nos précisions infra). Le numéro 10 de la revue Palimpsestes dédié à cette problématique, tout comme les diverses études actuelles sur la question rendent compte de la dynamique du phénomène et justifient la nécessité de l'approfondissement de son analyse dans les différentes langues, en fonction de la typologie discursive.

Après un bref rappel des problèmes d'ordre terminologique et taxinomique, nous allons discuter les principaux défis que suppose pour le traducteur le passage des caractéristiques du registre d'une langue dans une autre, avec une attention particulière aux paramètres de la situation d'énonciation et les marques du style d'un auteur (dans notre cas les versions roumaines du roman de Marguerite Duras, Moderato Cantabile). 


\section{Registre, niveau, style. Précisions d'ordre terminologique}

Autant du point de vue terminologique que taxinomique, la problématique des registres de langue est soumise à bien de controverses, plusieurs modèles étant proposés, selon les écoles et les approches; en termes de fréquence, dans le domaine français, le terme niveau est, conformément à $\operatorname{GADET}(1996,2007)^{1}$, le plus souvent utilisé, étant concurrencé par registre et style, et n'implique pas obligatoirement une hiérarchie, malgré des opinions encore présentes dans ce sens dans des ouvrages de spécialité; les termes interviennent de manière circulaire dans les définitions, servant comme notions tantôt génériques, tantôt spécifiques. Le Trésor de la langue française définit le niveau de langue comme une variété stylistique, que l'on distingue plus ou moins arbitrairement à l'aide de marques (d'ordre temporel, spatial et socio-culturel).

Reliant la langue à son contexte, donc passant obligatoirement par des critères d'ordre extra-linguistique, la notion de niveau devrait être un instrument utile pour surprendre autant les divergences entre les discours des différents locuteurs que celles qui particularisent les discours d'un même locuteur dans des situations de communication différentes. Si l'on se rapporte au modèle théorique traditionnel de la variation (diatopique, diachronique, diastratique, diaphasique), le niveau correspondrait, dans l'acception la plus courante à l'heure actuelle, à la variation diaphasique, qui prend en compte la dimension stylistique /situationnelle.

Le terme registre a des acceptions plus ou moins larges par rapport à niveau et style, servant soit comme terme général, soit comme terme spécifique. Associé à la langue ou bien, le plus souvent, au discours, le terme définit, selon le Trésor de la langue française, les usages de la langue ou du discours selon les milieux où on l'emploie ou selon les situations psychologiques dans lesquelles se trouve l'émetteur. Largement diffusé par les approches sociolinguistiques, il désigne une variété isolable d'une langue employée dans des situations sociales définies, sans que l'on puisse arriver cependant à une superposition parfaite entre les caractères sociaux et un ensemble de variables, les locuteurs ayant en général recours à plusieurs registres à la fois (cf. AUGER, 1997, in : CHARAUDEAU, MAINGUENEAU, 2002 : 495).

Dans le domaine anglo-saxon, bien des auteurs l'associent, à partir de Halliday, à la variation selon l'usage (qui s'oppose à la variation selon l'usager), son fonctionnement étant décrit en fonction de trois paramètres qui affectent et conditionnent la structure du discours envisagé, (la tripartition field/tenor/mode étant bien connue: selon le ton du discours (tenor), notamment la relation émetteur-récepteur telle qu'elle est envisagée dans le texte, on établit par exemple la dichotomie formel /informel; selon le canal (mode), on peut traiter de l'opposition oral vs écrit; selon le rôle du discours dans la transmission du message (field), on identifie par exemple des textes à caractère informatif, ou

\footnotetext{
${ }^{1}$ De manière intéressante, le syntagme niveau de langue apparaît assez tard, au milieu du $\mathrm{XX}^{\mathrm{e}}$ siècle, étant précédé par différents autres termes ou syntagmes, comme marque d'usage ; on l'impose autant pour des besoins strictement pédagogiques, en raison des rapports implicites, mais pas toujours clairs avec la norme, que traductologiques (voir l'intérêt de la stylistique comparée pour la question).
} 
marqués par la fonction émotive / conative / métalinguistique / phatique. (cf. BELL, 2000 : 27).

Le même manque de consensus caractérise le nombre des registres ou niveaux et le rapport qui s'institue entre ceux-ci (hiérarchique ou autre); l'énumération et la dénomination des étiquettes censées définir l'appartenance d'un mot ou d'une structure à une classe ou à une autre restent des démarches toujours problématiques. Il y a longtemps que les dictionnaires étiquettent les lexèmes de la langue selon des schémas plus ou moins complexes, sans pour autant arriver à construire un tableau systématique et unitaire de la répartition des registres du moins au niveau lexical. Puisque, dans l'analyse des registres, on envisage le plus souvent le plan lexical, les dictionnaires de référence pour une langue donnée sont un bon exemple pour illustrer le pullulement terminologique et taxinomique spécifique pour la problématique des registres. On hésite ainsi entre des modèles à trois, quatre et même cinq catégories, à partir de la variante standard, censée représenter le niveau non-marqué et simultanément le modèle à suivre, jusqu'au argotique, d'un côté de l'échelle, et au soutenu, de l'autre ${ }^{2}$, en passant par le familier et le populaire.

Ce qui rend plus délicate la tâche de l'étiquetage (du discours et du locuteur qui en est responsable) est le fait que la réalité des productions discursives, à laquelle les travaux sur des corpus attestés apportent toujours des éclaircissements importants, ne permet pas d'envisager le fonctionnement d'un registre ou d'un autre dans son intégralité, affectant tous les niveaux de l'analyse linguistique en même temps (lexique, morphosyntaxe, discours, et ainsi de suite); ainsi, un lexème familier n'a pas toujours pour correspondant une structure syntaxique du même registre :

«La distinction entre phénomènes marqués et non marqués permet aussi de montrer qu'il n’y a pas fonctionnement en conglomérats : par exemple, une négation sans $n e$, phénomène tellement répandu à l'oral qu'il ne saurait être considéré comme stigmatisant, est compatible avec une palette de variation dans l'une ou l'autre direction. Tout discours comporte des traits qui ne vont pas nécessairement dans la même direction, et qui réalisent un équilibre fragile et instable. La capacité d'évaluation du locuteur semble donc opérer de façon assez fine (même si elle est largement inconsciente), selon une globalisation qui n'est sûrement pas dichotomique » (GADET, 1996 : 29, c'est nous qui soulignons).

Pour ce qui est de la notion de style, souvent employée en relation à - ou comme synonyme de - niveau de langue, marque d'usage, on ne peut que rappeler ici les observations fort pertinentes de MAINGUENEAU (2002: 551): incontournable, la catégorie du style se trouve à la croisée de l'ensemble des sciences humaines, la stylistique ayant de nombreuses interférences avec l'analyse du discours, surtout là où elle permet de pousser la réflexion sur les relations entre les œuvres littéraires et leurs contextes. A notre avis, l'étude de la traduction littéraire y est implicitement incluse.

\footnotetext{
${ }^{2}$ Voir dans ce sens les remarques de GADET $(1996: 18)$ et nos propres observations (ARDELEANU et BALATCHI, $2005:$ 73).
} 


\section{Enjeux et stratégies de traduction des registres de langue}

Le but d'une traduction doit concerner, comme le montre Nida, non pas la reproduction de la forme d'un message mais la réponse que le récepteur du texte traduit est censé donner, et qui doit être comparable à celle des récepteurs du texte d'origine. Elle doit produire une «équivalence fonctionnelle», et c'est une question qui est toujours incluse dans l'établissement de la liste des «priorités » dressée pendant l'étape de préparation d'une traduction. Nida mentionne parmi ces priorités :

1. La correspondance contextuelle passe devant la correspondance verbale.

2. L'équivalence dynamique est plus importante que la correspondance formelle.

3. La forme orale de la langue est prioritaire par rapport à sa forme écrite.

4. La traduction doit envisager ce qui est utilisé et considéré comme acceptable par le public auquel elle s'adresse plutôt que ce qui est considéré comme prestigieux par la tradition.

Il s'agit donc de ne jamais perdre de vue la dimension contextuelle inhérente à toute production discursive, vu que la qualité de la traduction est toujours conditionnée par une interprétation pertinente du contexte. NIDA (2001) affirme dans ce sens que l'échec de compréhension des contextes est responsable dans la plupart des cas pour la mauvaise compréhension et traduction des discours. Par contexte, l'auteur souligne qu'il faut comprendre les aspects qui influencent, d'une part, les niveaux de production discursive (phonologique, lexical, grammatical) et, d'autre part, ceux qui ont trait à l' « histoire » du texte à traduire, notamment les événements précédant la production de ce texte, la façon dont il a été interprété, etc. C'est que les traducteurs ne traduisent pas des langues mais des textes.

Selon Nida, la traduction du style d'un texte est d'habitude regardée comme secondaire par rapport à son contenu ; un simple regard à travers les différentes approches montre que cette dimension compte, cependant, parmi les principes de traduction importants.

Dans l'histoire de la traductologie, on a toujours prêté une attention particulière à la question des registres, quel que soit le type d'approche (pédagogique, prescriptive ou au contraire descriptive). Bien des théories sur la traduction établissaient, parmi les «lois» absolues d'une bonne traduction la préservation du style de l'écriture du texte original (TYTLER, cité par BELL, 2000 : 29). Dans tout texte, le traducteur est tenu de rechercher et d'identifier ce que BELL (2000) appelle les indicateurs de la relation entre l'émetteur et le récepteur, le canal sélectionné pour la transmission du message et la fonction du discours.

Les «méthodes de traduction» accordent une place importante à la problématique des styles et registres, comme nous l'avons déjà remarqué (les titres de ces ouvrages étant eux-mêmes révélateurs, e.g. Stylistique comparée $d u$ français et de l'anglais, VINAY, DARBELNET). Les approches linguistiques et sociolinguistiques de la traduction soulignent l'importance de l'interprétation correcte de cette dimension du texte, qui doit précéder l'acte traductif en tant que tel, en se servant en principal des étiquettes présentes dans les dictionnaires (Chuquet et PAILlard, in: TencheA, 2008: 137). La recherche des 
correspondances possibles des registres est essentielle, divers types de compensation étant envisageables là où la distance entre les langues (vues surtout comme langes-cultures) ne le permet pas. (PEETERS, in : TENCHEA, 2008 : 137).

Pour ce qui est des stratégies de la traduction des registres, qui s'imposent dans le cas de la «non-correspondance des variétés de langue sociosituationnelles », CRISTEA (2000: 176), souligne qu'il est très délicat de faire équivaloir les variétés des deux langues telles qu'elles apparaissent dans les dictionnaires. L'interférence discursive peut toucher tous les niveaux de la variation, et influer sur la cohérence du texte traduit, avec des implications importantes du point de vue de l'équivalence culturelle. On aura par conséquent : des interférences diastratiques; des interférences diaphasiques ; des interférences diachroniques; des interférences diatopiques. Suite aux études sur des corpus de textes traduits en différentes langues, on a souligné qu'il est très fréquent d'avoir recours à une traduction explicative pour résoudre la question de la transposition des termes spécifiques à une aire d'utilisation. Un autre cas de figure peut être la neutralisation : les connotations socio-culturelles sont annulées à cause du choix de la variante non-marquée, ou bien elles peuvent être utilisées en vue d'une « compensation », là où d'autres marques sont impossibles à être rendues.

La compréhension et la connaissance du fonctionnement des registres dans les deux langues mises en contact par l'acte traductif sont des aspects constamment pris en ligne de compte dans les approches critiques de la traduction. Dans l'allocution d'ouverture du Colloque sur les niveaux de langue et registres en traduction, Bensimon affirmait que la confusion des niveaux de langue et/ou des registres est un des critères d'évaluation de la médiocrité d'une traduction, n'ayant d'égale que le flou méthodologique qui entoure la notion (cf. KOUSTAS, 1994 : 224). C'est pour cela que le bilinguisme et le biculturalisme des traducteurs doivent intégrer absolument la connaissance approfondie des niveaux de langue et registres de discours, que l'on a intérêt, surtout dans une optique traductologique, d'envisager par un rapport étroit avec la notion de connotation, vu l'infirmité de certains « style labels ». (idem, 225).

Par ailleurs, le rapport du registre au style doit être également pris en considération, très souvent le traducteur imposant son style au texte traduit, par le traitement particulier des coordonnées linguistiques et extra-linguistiques. Dans son analyse de la version italienne des Fleurs bleues de Raymond Queneau, réalisée par Italo Calvino, FEDERICI (2009) observait que les changements majeurs de Calvino en termes de registre et de style (par l'épuration des éléments dialectaux et régionaux) se fondent sur des connaissances poussées en linguistique et sur sa façon particulière de relier ce qui est transmissible à ce qui est traduisible.

Au-delà de la nécessité inhérente de la connaissance et reconnaissance des particularités de registre, niveau et style de chaque langue, toute analyse de cette problématique doit également prendre en compte le côté essentiellement subjectif de l'acte traduisant, bien des choix se justifiant uniquement par rapport à la manière personnelle d'interprétation du texte du traducteur. Comme le souligne HEWSON (1996 : 89):

«Le traducteur n'est pas un opérateur neutre, mais un individu, avec à la fois son histoire linguistique et culturelle, sa compétence linguistique et sa propre conception de la traduction. Chaque acte de traduction implique un positionnement par rapport aux deux langues-cultures en 
présence, où l'on peut essayer de privilégier les caractéristiques linguistiques, voire idéologiques, de la langue de départ, ou bien de les normaliser au maximum. »

\section{Moderato Cantabile en roumain. Traduction et retraduction du style et registre(s)}

Lorsqu'on passe de la théorie à la pratique, on se rend compte qu'il est toujours beaucoup plus facile d'énoncer ce que le traducteur doit accomplir que de produire de manière effective un texte dans la langue cible respectueux à tous les niveaux du registre de la langue source.

La traduction des textes littéraires est particulièrement sensible à la problématique des registres. Dans le roman Moderato cantabile de Marguerite Duras, le registre soutenu, recherché, bien marqué au niveau du récit se mêle en permanence au registre standard et au registre familier, informel, qui ponctuent constamment les dialogues rapportés en style direct et les discours rapportés en style indirect. Ces interférences sont différemment rendues dans les deux versions du roman en roumain que nous allons prendre ici comme base de discussion, notamment la traduction de Alexandru Baciu ${ }^{3}$ (Moderato cantabile, Editions Albatros, 1974) et une traduction plus récente, qui appartient à une jeune écrivaine et rédactrice, Carmelia Leonte (Moderato cantabile, Editions Cartier, 2006). La distance d'à peu près trente ans qui sépare les deux versions devrait mettre sous un jour plus favorable la nouvelle traduction, qui a normalement comme but de récréer un texte, en améliorant, implicitement ou explicitement, la version existante ${ }^{4}$. Nous allons voir à quel point cette raison d'exister d'une nouvelle version se manifeste au niveau de l'écriture et d'un style tellement particuliers, des choix dans la gamme des structures marquées/ non-marquées des registres.

C'est au niveau de la traduction des dialogues que nous pouvons faire des remarques assez intéressantes au sujet des stratégies de transposition des registres, les problèmes surgissant au niveau des particularités de la situation d'énonciation. Nous allons nous arrêter sur quelques exemples de dialogue entre la mère / l'enfant et la professeure de piano. Il s'agit donc d'une situation de communication orale, rendue à travers le discours en style direct, où le registre standard est constamment parsemé d'intrusions du registre familier, engendrées par : le type de relation interpersonnelle (familiale, donc plutôt relâchée entre la mère et l'enfant ; professionnelle, ce qui veut dire plus contraignante entre l'enfant et sa professeure et la mère et la professeure); les particularités de la situation de communication en tant que telle, (au fil d'une interaction orale au départ «contrôlée » par les intervenants, il arrive que la conversation se détende et reçoive l'empreinte plus marquée de la familiarité et de l'oralité).

C'est que la notion de contexte elle-même doit être perçue, comme le soulignent bien les tenants de la théorie de la pertinence, SPERBER et

\footnotetext{
${ }^{3}$ Traducteur, écrivain et rédacteur. Il traduit en roumain une vingtaine d'ouvrages de littérature et philosophie française, dont notamment Marguerite Duras, Jean Giono, Alain (en collaboration avec Nicolae Steinhardt).

${ }^{4}$ Dans leur ouvrage sur la retraduction, MONTI et SCHNYDER (2011:6) observaient que, parmi les raisons qui justifient le phénomène, comptent essentiellement les besoins d'actualiser, d'améliorer, ou encore de corriger.
} 
WILSON (1989) en tant que dynamique et non pas statique, s'adaptant aux besoins conversationnels. Les relations entre les participants peuvent évoluer au fil de la même interaction verbale: la politesse qui caractérise les interactions entre la mère et la professeure par exemple fond de temps en temps sous la tension des répliques. C'est ce jeu bien subtil qui doit être surpris lors de la traduction; on rencontre cependant souvent des solutions approximatives et même ratées dans les versions roumaines à cause justement de la mauvaise compréhension du contexte communicationnel.

Le tout début du roman nous fait entrer de plain-pied dans un dialogue «didactique », avec une interrogation à valeur de requête de la part de la professeure, construite selon les règles du français standard; dans la version de Baciu, l'interrogation préserve son caractère relativement neutre, le présent du verbe roumain a vrea [vouloir] ayant le même rôle de construire une requête à valeur d'ordre implicite: vrei să citeşti [veux-tu lire]; cette interrogation est traduite par Leonte d'une manière nettement plus marquée au niveau de la politesse [as-tu la bonté de lire...], ce qui construit déjà un autre type d'attente, pour le lecteur, vis-à-vis de la relation interpersonnelle, qui sera contredite par la partie ultérieure du texte :

(1) - Veux-tu lire ce qu'il y a d'écrit au-dessus de ta partition? demanda la dame.

- Vrei să citeşti ce scrie deasupra partiturii ? întrebă profesoara (traduction A.B.)

- $\quad$ Eşti bun să citeşti ce scrie pe partitură ? întrebă doamna.(traduction C. L.)

$\mathrm{Au}$ niveau des termes qui désignent les personnages et qui seront utilisés également comme termes d'adresse le long du texte, la distance temporelle qui sépare les deux traductions (une trentaine d'années) justifie peut-être le choix différent, chaque traducteur optant pour le terme qu'il considère le mieux adapté au contexte socioculturel de la réception du texte (profesoara [la professeure] vs doamna [la dame]).

Les interrogations avec structures emphatiques portant sur différents constituants de la phrase sont un autre aspect qui relève de la dimension du registre. Ainsi, pour traduire les structures avec détachement et reprise, spécifiques du discours oral, les deux versions optent pour des solutions différentes au cas de l'interrogation partielle suivante, avec annonce du sujet par le démonstratif cataphorique ça:

(2) - Et qu'est-ce que ça veut dire, moderato cantabile?

- Şi ce-nseamnă asta, moderato cantabile ? (traduction A.B.)

- $\quad$ Şi ce înseamnă moderato cantabile ? (traduction C. L.)

Si toute marque de registre familier est annulée dans la version de Leonte, la version de Baciu la préserve et la renforce autant par le choix de la forme familière du démonstratif neutre roumain, asta, $[c ̧ a]$ que par l'élision de l'initiale vocalique de la forme verbale, particulièrement fréquente dans la variante orale, familière de la langue, assurant un débit plus rapide.

Le jeu des modalités d'énonciation et d'énoncé pose d'ailleurs des problèmes à plusieurs points dans le texte de Leonte, la non-compréhension du contexte 
énonciatif engendrant même des traductions erronées, comme dans les deux exemples ci-dessous :

(3)-Madame Desbaresdes, quelle tête vous avez là, dit-elle.

Anne Desbaresdes soupira une nouvelle fois.

- A qui le dites-vous?

- Doamnă Desbaresdes, ce copil încăpățânat aveți! - îi zise.

Anne Desbaresdes oftă din nou :

- Mie-mi spuneți! [A.B.]

- Doamnă Desbaresdes, ce spuneți, zise ea.

Anne Desbaresdes a suspinat încă o dată.

- $\quad$ Ce părere aveți ? [C. L.]

L'exclamation de la professeure de piano, quelle tête vous avez là, qui fonctionne, dans le contexte, comme un acte de reproche adressé à la mère à propos de l'entêtement de son enfant, est rendue par un énoncé à valeur pragmatique similaire dans la version de Baciu, à travers une explicitation (quel enfant entêté vous avez), la réplique de la mère étant également parfaitement correspondante sur le plan pragmatique, malgré la substitution de la modalité interrogative par la modalité exclamative (Mie-mi spuneți! [C'est à moi que vous le dites!]). La non-compréhension de ce même énoncé initial mène à une adaptation du dialogue, assez incohérente, dans la version de Leonte, qui élimine complètement l'exclamation et la mention de l'entêtement de l'enfant, en les remplaçant par une interrogation qu'est-ce que vous dites, et en enchaînant le dialogue par une autre, quasiment synonyme : quelle est votre opinion pour à qui le dites-vous?

(4)-Je ne veux plus qu'on te gronde, sans ça, j'en meurs.

- Nu mai vreau să te ocărască, altfel să ştii c-am să mor. [A.B.]

- Nu vreau să te cert, dar simt că e prea de tot. [C. L.]

Le verbe gronder, auquel le Petit Robert assigne l'étiquette familier, est traduit par la variante neutre a certa (l'équivalent roumain de réprimander) chez Leonte, annulant ainsi toute marque de registre, et par le verbe a ocărî chez Baciu, variante présentée comme populaire par le dictionnaire de référence pour le roumain, DEX, ce qui crée une image du personnage, de son appartenance sociale, des particularités de son discours et de la relation interpersonnelle sensiblement différente par rapport à l'original. La traduction du pronom on s'avère problématique, dans la mesure où Leonte l'associe, de manière erronée, à la première personne (je ne veux pas te gronder). La deuxième partie de la phrase, toujours marquée par l'oralité est également rendue de manière différente dans les deux traductions : une structure parallèle correcte chez Baciu, avec une addition nécessaire pour la cohérence de l'énoncé (sinon, sache que je vais mourir) et le remplacement du présent par le futur périphrastique familier du roumain, (je vais mourir), peut-être aussi par besoin de compensation du caractère familier, oral du dialogue, effet créé également par l'élision de la conjonction de subordination que (că), phénomène toujours caractéristique de l'oral (c-am să mor). Dans la version de Leonte, la distance et la liberté que prend la traductrice par rapport à l'original, proposant mais je sens que c'est trop pour sans ça, j'en meurs n'est justifiée en 
aucun cas par le contexte discursif, créant des écarts sémantiques et énonciatifs importants, qui brouillent la réception de la relation interpersonnelle.

Les techniques de préservation ou de compensation dans la traduction des marques du niveau oral, familier de l'interaction se heurtent à des difficultés accrues lorsqu'il s'agit de transposer les interrogations et plus généralement les énoncés réduits au GN, pour lesquels il semble que le français ait un goût bien marqué ${ }^{5}$. Dans les deux versions roumaines on a choisi de reconstituer la phrase canonique, les traducteurs proposant des phrases avec sujet et verbe prédicatif actualisé :

(5) - Quelle mesure, cette jolie petite sonatine?

- Ce măsură are această mică şi drăguță sonatină ? [A.B.]

- Care-i măsura pentru această micuță şi drăguță sonatină? [C. L.]

(6) -Des autos qui arrivent, dit-il.

- Sosesc maşini, anunță el. [A.B.]

- Vin nişte maşini, spuse el. [C. L.]

Toutefois, dans le cas de l'interrogation (exemple 5), la traduction de Leonte est mieux marquée au niveau du registre familier, par le choix de la forme élidée du verbe être, ce qui accentue l'oralité de l'énoncé. Nous pensons que l'on aurait pu compenser la perte au niveau de l'ellipse du verbe par le choix de la forme familière du démonstratif cette - asta au lieu de la forme standard aceasta. De même, il n'y a pas lieu, selon nous, de procéder à une antéposition des adjectifs qualificatifs mică [petite] et drăguţă [jolie], normalement postposés en roumain dans des situations non-emphatiques (Care-i măsura pentru sonatina asta mică şi drăguță ?), situation qui caractérise le contexte de l'énoncé original aussi. Pour ce qui est de l'énoncé assertif de l'exemple 6, où l'ellipse d'un prédicat comme voilà rend très bien compte de cette «immédiateté » des impressions de l'enfant, nous préférons encore une fois la solution de Leonte, le verbe venir, à la différence d'arriver, que propose Baciu, semblant beaucoup plus naturel dans la bouche d'un enfant et ayant aussi l'avantage de la brièveté au présent de l'indicatif.

\section{En guise de conclusion}

Si, comme le souligne GADET (1996 : 35), le seul travail utile dans le domaine de la variation en langue réside dans l'exploration des dimensions proprement linguistiques, il est essentiel, pour le traducteur, d'avoir une connaissance approfondie des contraintes qui régissent les traits des niveaux dans chacune des deux langues mises en contact dans la dynamique du processus traductif.

L'interférence des registres reste, pour tout traducteur, un problème délicat. Une bonne compréhension des paramètres contextuels et en particulier de la relation interpersonnelle des participants à la situation de communication est une condition sine qua non de l'activité de traduction; dans la mesure où toute traduction implique une réécriture, compte tenu des spécificités de la langue cible

\footnotetext{
5 «Le tour appartient sans doute à un registre plus moderne, mais il semble plus naturel et plus séduisant dans le registre oral, plus élégant et plus léger dans le registre écrit, où il permet également d'exprimer avec plus de force et d'immédiateté des émotions ou des impressions » (BALLARD, 1996 : 202).
} 
et du fonctionnement des registres dans le nouveau contexte de la traduction, traduire le registre équivaut finalement à un exercice de style, qui est de manière inhérente, soumis à la subjectivité du traducteur ${ }^{6}$.

\section{BIBLIOGRAPHIE}

\section{Ouvrages de référence}

ARDELEANU Sanda-Maria; BALATCHI Raluca-Nicoleta (2005), Eléments de syntaxe du français parlé, Iaşi, Institutul European.

BALATCHI Raluca-Nicoleta (2007), L'expression de la subjectivité dans le langage, Iaşi, Demiurg.

BALLARD Michel (1996), Enoncés sans verbes et registres en traduction, Palimpsestes, no. 10, Niveaux de langue et registres de la traduction, Paris, Presses de la Sorbonne Nouvelle, p. 179-203.

BELl Roger (2000), Teoria şi practica traducerii, Iaşi, Polirom (trad. de C. Gazi).

BERMAN Antoine (1995), Pour une critique des traductions : John Donne, Paris, Gallimard.

Charaudeau Patrick; MaIngueneau Dominique (2002), Dictionnaire d'analyse du discours, Paris, Seuil.

CONSTANTINESCU Muguraş ; STEICIUC Elena-Brânduşa (coord.) (2011), Atelier de traduction, no. 15 et 16, Dossier: La traduction caduque, retraduction et contexte culturel (en diachronie), Suceava, Editura Universitătii.

CRISTEA Teodora (2000), Stratégies de la traduction, București, Editura Fundației România de Mâine .

FEDERICI F. (2009), Translation as stylistic evolution, Amsterdam, Rodopi.

GADET Françoise (1996), Niveaux de langue et variation intrinsèque, Palimpsestes, no. 10, Niveaux de langue et registres de la traduction, Paris, Presses de la Sorbonne Nouvelle, p. 17-41.

GADET Françoise (2007), La variation sociale en français, Paris, Ophrys.

HEWSON Lance (1996), Le niveau de langue repère, Palimpsestes, no. 10, Niveaux de langue et registres de la traduction, Paris, Presses de la Sorbonne Nouvelle, p. 77-93.

KAHN Robert ; SETH Catherine (éds.) (2010), La Retraduction, Publications des Universités de Rouen et du Havre.

Koustas Jane (1994), Niveaux de langue et registres en traduction, compterendu, TTR : traduction, terminologie, rédaction, vol. 7, no. 2, p.224-228.

MULLER M. S., (1996), Langue familière, parler populaire, particularisme régional dans Saturday Night and Sunday Morning d'Alan Sillitoe et sa traduction française, Palimpsestes, no. 10, Niveaux de langue et registres de la traduction, Paris, Presses de la Sorbonne Nouvelle, p. 49-77.

NIDA Eugene (2001), Contexts in Translating, Amsterdam, John Benjamins.

NIDA Eugene; TABER C. (1969), The Theory and Practice of Translation, Amsterdam, Brill.

SPERBER Dan; WILSON Deirdre (1989), La pertinence, Paris, Minuit.

\footnotetext{
${ }^{6}$ Cet article est le résultat d'une recherche effectuée dans le cadre du projet PNCDI, CNCSUEFISCDI, PN-II-RU-PD-2011-3-0125.
} 
TENCHEA Maria (2008), Dicționar contextual de termeni traductologici, Timişoara, Editura Universităţii de Vest.

TRABELSI C. (2000), La traduction des niveaux de langue et des régionalismes de l'arabe en français dans le roman de Taïeb Salah, Saison de la Migration vers le Nord, Meta : Journal des traducteurs / Meta: Translators' Journal, vol. 45, $\mathrm{n}^{\circ} 3$, p. 465-474.

VINAY Jean-Paul ; DARBELnET Jean (1966), Stylistique comparée du français et de l'anglais, Paris, Gallimard, 1966.

\section{Dictionnaires}

DEX, Dicționarul explicativ al limbii române (1998), Univers Enciclopedic, Bucureşti.

Le Petit Robert. Dictionnaire alphabétique et analogique de la langue française, (2001), Dictionnaires Le Robert, Paris.

Trésor de la langue française informatisé, http://atilf.atilf.fr/

\section{Corpus d'analyse}

DURAS Marguerite (1958), Moderato Cantabile, Paris, Minuit.

DuRAs Marguerite (1974), Moderato Cantabile, trad. Alexandru Baciu, Bucureşti, Albatros [A.B.].

DuRAS Marguerite (2006), Moderato Cantabile, trad. Carmelia Leonte, Chişinău, Cartier [C.L.]. 